\title{
Online Editorial Management-Systeme und die Produktion wissenschaftlicher Fachzeitschriften ${ }^{1}$
}

\section{Einleitung}

Mit den Begriffen »cyberinfrastructure $«^{2}$, »cyberscience $«^{3}$ und $»$ e-Research $\aleph^{4}$ wurden wir in jüngerer Vergangenheit immer wieder darauf aufmerksam gemacht, dass die zunehmende Nutzung von Computernetzwerken und auf dem Internet basierender Medien auch für die Wissenschaft einen großen Wandel bedeutet. Dieser Wandlungsprozess betrifft nicht nur die Forschung selbst, sondern die Wissenschaft in umfassenderem Sinne. Auch im Kernbereich des formalen wissenschaftlichen Kommunikationssystems, in den Redaktionen wissenschaftlicher Fachzeitschriften, wurden mit sogenannten Online Editorial Management-Systemen bei vielen Zeitschriften Infrastrukturen eingeführt, mit denen die Produktion wissenschaftlicher Journale verändert wird. Diese Veränderung bezieht sich auf den gesamten Prozess: von der Manuskripteinreichung über die Begutachtung und Überarbeitung bis hin zur Fertigstellung von Druckvorlagen. Der vorliegende Artikel geht der Frage nach, welche Veränderungen der herausgeberischen Arbeit mit der Verwendung dieser Systeme verbunden sind. Meine These lautet dabei, dass mit der Einführung von Online Editorial Management-Systemen eine Form von Arbeitsorganisation und Kontrollstruktur geschaffen wird, die sonst für formale Organisationen typisch ist. Bei der Produktion von Zeitschriftenartikeln sind nun aber nicht nur Verlage, sondern zu einem erheblichen Teil Mitglieder wissenschaftlicher Fachgemeinschaften beteiligt - sei es in der Rolle des Autors, des Gutachters oder sei es in der Rolle des Herausgebers. Arbeitsorganisation und Kontrollstruktur werden gewissermaßen über die Grenze der Organisation »Verlag « ausgedehnt und in die wissenschaftliche Fachgemeinschaft hineinverlagert. Wie zu zeigen ist, führt dies zu Zusammenstößen zwischen ökonomischer und wissenschaftlicher Rationalität.

Die These wird in vier Schritten entwickelt. Zunächst wird die Datengrundlage vorgestellt, auf die sich die Argumentation stützt: meine Tätigkeit als Managing Editor der Zeitschrift Minerva. In einem zweiten Schritt wird in Absetzung zum üblichen wissenschaftssoziologischen Blickwinkel eine Produktionsperspektive auf

1 Für Hinweise danke ich Uwe Schimank, den Teilnehmern der Ad-hoc-Gruppe »Die Zukunft des wissenschaftlichen Publizierens « auf dem Kongress der Deutschen Gesellschaft für Soziologie 2010 sowie zwei anonymen Gutachtern des Leviathan.

2 Atkins et al. 2003.

3 Nentwich 2003.

4 Jankowski 2009. 
wissenschaftliche Fachzeitschriften entworfen. Hieran schließt sich in einem dritten Schritt die Vorstellung eines Online Editorial Management-Systems an, das hinsichtlich der Dimensionen » Arbeitsorganisation « und »Kontrollstruktur « analysiert wird. In einem abschließenden Schritt geht es viertens darum, herauszuarbeiten, welche Art von Kollisionen zwischen wissenschaftlicher und ökonomischer Rationalität durch die Nutzung derartiger Systeme provoziert wird.

\section{Datengrundlage}

Die folgende Analyse basiert auf meiner Arbeit als geschäftsführender Herausgeber der internationalen Fachzeitschrift Minerva, deren Redaktion am Institut für Wissenschafts- und Technikforschung (IWT) an der Universität Bielefeld beheimatet ist. Sie erscheint vierteljährlich und wird herausgegeben von Peter Weingart, der von einem internationalen Beratergremium unterstützt wird. ${ }^{5}$ Eine Besonderheit der Zeitschrift besteht in ihrer interdisziplinären Konzeption. Indem sie regelmäßig Beiträge aus den Bereichen Wissenschaftsgeschichte, Wissenschaftssoziologie, Wissenschaftsphilosophie sowie aus der Innovationsforschung und Hochschulforschung publiziert, bemüht sie sich um einen Brückenschlag zwischen wissenschaftsreflexiven Forschungsgebieten, die verschiedene disziplinäre Ursprünge haben.

Die Eigentumsrechte am Journal liegen bei Springer Science+Business Media, einem Verlag, der laut seiner Webseite neben Minerva etwa 2.000 weitere Journale und mehr als 50.000 E-Books verlegt. ${ }^{6}$ Mit diesen sowie mit Lehrbüchern, Sachbüchern, Handbüchern, Reihen und Atlanten setzte der Verlag im Jahr 2009845 Mio. Euro um. ${ }^{7}$ In dieser Größenordnung ist das Geschäft mit wissenschaftlichen Publikationen auch für Finanzinvestoren interessant, wie die Besitzverhältnisse am Springer-Verlag zeigen: Seit 2009 ist Springer anteiliges Eigentum der privaten Investmentgruppe EQT und der Government of Singapore Investment Corporation (GIC) ${ }^{8}$

Mit dem zweiten Heft des Jahres 2008 hat die Herausgeberschaft von Minerva von Roy MacLeod zu Peter Weingart gewechselt, und seit diesem Zeitpunkt bin ich als Managing Editor für die Organisation der Begutachtung und für den Schriftverkehr mit den Autoren verantwortlich. Die Bielefelder Redaktion nahm die Arbeit Mitte des Jahres 2007 auf, um im Juni 2008 ihr erstes Heft vorlegen zu können. Mit dem Wechsel der Herausgeberschaft wurde zwischen Springer und dem neuen Editor vereinbart, das Online Editorial Management-System Editorial Manager einzu-

5 Die Zusammensetzung des Beratergremiums findet sich auf der Webseite von Minerva unter: http://www.springer.com/education+\%26+language/journal/11024 (Zugriff vom 03.04.2012).

6 Siehe http:/www.springer.com/about+springer/company+information/key+facts?SGWID =1-175806-0-0-0 (Zugriff vom 03.04.2012).

7 Springer 2009.

8 Vgl. Börsenblatt vom 11. Dezember 2009, http://www.boersenblatt.net/350274/ (Zugriff vom 03.04.2012). 
führen. Seit September 2007 wird dieses System nun verwendet, und bis heute wurden mehr als 300 Papiere darüber eingereicht, deren Begutachtung über das System organisiert wurde.

Die hier präsentierten Ergebnisse basieren also auf einer mehrjährigen mehr oder minder täglichen Arbeit mit dem System, die auch die Phase der Einführung mit einschließt. Was die Methode der Datenerhebung und -analyse angeht, lässt sich von einer intensiven teilnehmenden Beobachtung sprechen. Beobachtungsphasen wechselten sich mit Phasen ab, in denen mit dem System gesammelte Erfahrungen reflektiert wurden. Dabei ergaben sich auf der Grundlage solcher Reflexion weitere Fragen, die im Umgang mit dem System beobachtungsleitend gewirkt haben. Für die Analyse erwies es sich als hilfreich, dass sämtliche über das System stattfindende Kommunikation protokolliert wurde. Diese automatische Protokollierung ermöglichte es, die Kommunikation zu einem späteren Zeitpunkt auszuwerten. Als besonders erkenntnisfördernd erwiesen sich spontan entstehende Eindrücke von Störungen der Interaktion, da sie Anlass boten, der Frage nachzugehen, welche Art von Problemen im Umgang mit dem System hier stattgefunden hatten und wodurch sie verursacht worden waren. Neben der Reflexion der Erfahrungen mit dem System fließen in die Analyse aber auch Erkenntnisse mit ein, die im Zuge von mehreren Treffen mit den für das Journal zuständigen Vertretern des Verlags gewonnen wurden. Gegenstand dieser Treffen waren unter anderem strategische Fragen zur Ausrichtung des Journals. Bei diesen Besprechungen wurde intensiv auf das System Editorial Manager und die vom System gesammelten Daten rekurriert.

\section{Eine Produktionsperspektive auf wissenschaftliche Journale}

Bevor die Auswirkungen von Online Editorial Management-Systemen auf die Produktion wissenschaftlicher Journale analysiert werden, sollen in diesem Schritt einige die Analyse anleitende theoretische Überlegungen angestellt werden. Innerhalb der Wissenschaftssoziologie werden wissenschaftliche Journale gemeinhin mit Blick auf ihre Funktion für die Wissenschaft - die Prüfung von Wahrheitsansprüchen und die Verbreitung von Forschungsergebnissen - diskutiert. Diesem Gegenstandszuschnitt nach ist das Fachjournal Teil des formalen wissenschaftlichen Kommunikationssystems und als solches innerhalb des sozialen Systems » Wissenschaft « situiert. Dies gilt für die klassischen Untersuchungen in der Tradition der Merton'schen Wissenschaftssoziologie ${ }^{9}$, die Erforschung des Begutachtungsprozesses ${ }^{10}$ und die Beforschung von Entscheidungsprozessen in den Redaktionen wissenschaftlicher Fachzeitschriften ${ }^{11}$. Nicht in den Blick kommt im Rahmen dieser in der Wissenschaftssoziologie gut verankerten Perspektive, dass die Produktion und die Verbreitung wissenschaftlicher Publikationen Vorgänge sind, die ein Zusammenwirken von sowohl wissenschaftlichen als auch nichtwissenschaftlichen Akteuren einschließen.

9 Siehe z.B. Zuckermann, Merton 1971.

10 Vgl. z.B. Peters, Ceci 1982; Hirschauer 2002.

11 Vgl. z.B. Hirschauer 2005.

Leviathan, 40. Jg., 2/2012 
Daher ist die gemeinhin von der Wissenschaftssoziologie angebotene Perspektive kaum geeignet, einen angemessenen Zugriff auf den Bereich des Sozialen zu gewinnen, innerhalb dessen Online Editorial Management-Systeme eingeführt werden.

Nach dem bisher Gesagten lassen sich die folgenden Anforderungen für eine adäquate Theorieperspektive formulieren: Zum einen sollte sie einen begrifflichen Rahmen bereitstellen, um grundlegende Akteursorientierungen erfassen zu können. Entscheidend sind hier der ökonomische und wissenschaftliche Orientierungshorizont. Zum anderen - und diese Anforderung ist für das Verständnis des Gegenstands mindestens ebenso wichtig - sollte die Theorieperspektive über einen starken Akteursbegriff verfügen und begriffliche Werkzeuge für die Analyse von Akteurskonstellationen liefern. Diese zweite Anforderung ist entscheidend zum Verständnis der Arbeitsorganisation und der Kontrollstruktur, die durch Online Editorial Management-Systemen geschaffen werden.

Die Anforderung nach einer Integration von Akteurs- und Differenzierungstheorie wird durch eine differenzierungstheoretisch informierte Lesart des akteurszentrierten Institutionalismus gut erfüllt. ${ }^{12}$ In Anschluss an die Arbeiten von Renate Mayntz und anderen lassen sich grundsätzlich drei Strukturdimensionen unterscheiden. Die oberste Ebene bildet die Differenzierung der Gesellschaft in funktionale Teilsysteme. Diese werden hier nicht als "gesellschaftsweit institutionalisierte, funktionsspezifische Handlungszusammenhänge ${ }^{13}$ verstanden, sondern als » hochgradig generalisierte sinnhafte Orientierungen, die den Akteuren als allgemein verbreitete situationsdefinierende Fiktion gegenwärtig sind « ${ }^{14}$ Von einer Fiktion ist zu sprechen, weil erhebliche Diskrepanzen zwischen den generalisierten Orientierungen einerseits und der Kontingenz konkreter sozialer Situationen andererseits bestehen und die Akteure sich dieser Diskrepanzen durchaus gewahr sind, sich aber dennoch an diesen simplifizierenden Abstraktionen orientieren. ${ }^{15}$ Die teilsystemischen Orientierungshorizonte sorgen für eine Reduktion von Komplexität, indem sie für das soziale Handeln von Akteuren einen Bezugsrahmen bereitstellen, der bestimmt, worum es in einer bestimmten Situation überhaupt geht. Dem Akteur liefern die Horizonte eine Orientierung dabei, in welche Richtung sein Wollen streben kann. ${ }^{16}$ Die zweite Strukturdimension bilden Akteurskonstellationen. Diese Ebene konzeptualisiert die grundlegende, aus der interaktionistischen Soziologie stammende Einsicht, dass Akteure ihre Interessen nicht monologisch vertreten können, sondern soziales Handeln immer im Zusammenspiel mit anderen Akteuren erfolgt, die ihrerseits handelnd Interessen verfolgen. Akteurskonstellationen bilden dabei für den Akteur nicht nur Restriktionen bei der Verfolgung von Interessen. Vielmehr wirken sie auch handlungsermöglichend, wenn beispielsweise Akteure mit komplementären Interessen

12 Aus diesem Grund wird der Theorie hier der Vorzug gegeben gegenüber der weiter ausgearbeiteten Alternative der Systemtheorie Luhmann'scher Provenienz.

13 Mayntz 1988, S. 17.

14 Schimank 2007, S. 220.

15 Schimank 1988, S. 632 f.

16 Schimank 2007, S. 220. 
Koalitionen bilden und so Ziele zu erreichen imstande sind, die sie allein nicht realisieren könnten. In dieser Strukturdimension geht es daher um das Können von Akteuren. ${ }^{17}$ Weiter lässt sich unterscheiden zwischen Beeinflussungs- Verhandlungs- und Beobachtungskonstellationen, wobei im Folgenden vor allem der letztgenannte Typ interessiert. Beobachtungskonstellationen sind dadurch gekennzeichnet, dass Akteure sich wechselseitig wahrnehmen und ihr Handeln durch den Umstand dieser wechselseitigen Beobachtung mit bestimmen lassen. ${ }^{18}$

Die letzte Strukturdimension bilden institutionelle Ordnungen, also gesellschaftliche Sollens-Vorgaben, die sich an Akteure richten. Diese normativen Strukturen können nicht nur unterschiedliche Reichweiten besitzen, sondern kommen auch in verschiedener Form vor. Zu ihnen zählen informelle Regeln wie Umgangsformen, also Sitte und Gebräuche, formalisierte Rechtsnormen und Verfahrensregeln oder auch Mitgliedschaftserwartungen von Organisationen. ${ }^{19}$

\subsection{Wissenschaftler als Autoren, Gutachter und Herausgeber}

Mit diesen etwas grundsätzlicheren Überlegungen ist das Handwerkszeug komplett, um ein Modell der Produktion wissenschaftlicher Journale zu entwickeln. Folgt man den Überlegungen von Hanekop und Wittke, ${ }^{20}$ kann man Akteurskonstellationen, die Fachzeitschriften hervorbringen, unter wirtschaftlichen Gesichtspunkten als eine Wertschöpfungskette betrachten, an der Akteure beteiligt sind, die sich an verschiedenen teilsystemischen Sinnhorizonten orientieren: Dort sind die Wissenschaftler, die in der Rolle des Autors Text produzieren und als Herausgeber eines Journals für die Akquise von Manuskripten sorgen, Gutachter auswählen sowie Entscheidungen über die Publikationswürdigkeit der eingereichten Beiträge treffen. In der Funktion des Gutachters kritisieren sie die Manuskripte, geben Hinweise zur Verbesserung der Verständlichkeit der Argumentation und beraten die Herausgeber bei ihren Entscheidungen. Mit der zugrunde gelegten Perspektive der Wertschöpfungskette machen Hanekop und Wittke für die Gruppe der Wissenschaftler eine Anomalie aus: Wissenschaftler sind als Autoren, Gutachter und Herausgeber an monetären Ressourcenflüssen nicht oder nur sehr eingeschränkt beteiligt und erbringen die genannten Leistungen meist freiwillig und unbezahlt oder werden nur gering ent-

17 Ebd. S. 221.

18 Schimank 2005, S. 33.

19 Schimank 2007, S. 221.

20 Hanekop, Wittke 2006. Im Folgenden wird auf eine im Zusammenhang der Reflexion von Open Access entstandene Perspektive auf das wissenschaftliche Publikationssystem zurückgegriffen. Dieser Rückgriff ist keineswegs zufällig, da das Verständnis der Entwicklung hin zum frei zugänglichen Publizieren nach einem anderen Gegenstandszuschnitt verlangt als die übliche wissenschaftssoziologische Perspektive. Dieser ist typischerweise umfassender und inkludiert Wissenschaftler, Verlage und Bibliotheken sowie deren Zusammenwirken. Siehe hierzu auch beispielhaft die ökonomisch inspirierten Überlegungen von Brintzinger 2010. 
lohnt. ${ }^{21}$ Die Bereitschaft, Beiträge im Rahmen der Wertschöpfungskette zu erbringen, lässt sich vor allem durch die Orientierung der Akteure am teilsystemischen Sinnhorizont ${ }^{22}$ der Wissenschaft erklären: Die Lieferung von Beiträgen zu den Debatten des Fachs erklärt sich durch das Streben der Wissenschaftler nach der Anerkennung durch Fachkollegen. ${ }^{23}$ Den Herausgebern kann eine ähnliche Orientierung unterstellt werden, da entsprechende Positionen - zumindest im Fall von renommierten Zeitschriften - Reputation versprechen. Etwas anders verhält es sich mit der Leistung der Gutachter, deren Beitrag zum Produktionsprozess die folgende Auffälligkeit zeigt: Gutachter stehen nach der Beauftragung zur Begutachtung eines Manuskripts unter »Lektürezwang «. ${ }^{24} \mathrm{Da}$ die Begutachtung in aller Regel in anonymer Form erfolgt, kann die Leistung des Gutachters nicht mit Reputation entgolten werden, so dass Reputationsgewinn als mögliches Handlungsmotiv ausfällt. ${ }^{25}$ Die Erbringung von Leistungen kann daher nur normativ mit Verweis auf institutionelle Ordnungen der Wissenschaft wie der Verpflichtung von Wissenschaftlern zur Kritik, ${ }^{26}$ die Sorge um die Qualität der Forschung in einem Fach ${ }^{27}$ oder das commitment gegenüber einer Zeitschrift und seinen Herausgebern erklärt werden.

\subsection{Verlage}

Innerhalb der Wertschöpfungskette produzieren Wissenschaftsverlage selbst keinen Inhalt (»content «), sondern stellen nur das Medium bereit, wie es für Medienproduzenten durchaus üblich sein kann. ${ }^{28}$ Sie nutzen also die Arbeit der Wissenschaftler, um ihre Verwertungsinteressen daran anzuschließen. Um dies zu realisieren, haben sich Verlage an zwei unterschiedlichen teilsystemischen Horizonten - nämlich der wirtschaftlichen und der wissenschaftlichen Rationalität - zu orientieren. Ökonomische Gewinne können für Wissenschaftsverlage nur dann realisiert wer-

21 Ebd., S. 204.

22 Schimank 2005.

23 Hagstrom 1965, S. 16.

24 Hirschauer 2005, S. 58.

25 Die einzige Möglichkeit, Gutachtertätigkeit in Reputation umzumünzen, besteht in deren Erwähnung im Lebenslauf.

26 Merton (1973 [1942], S. 277 f.) bezeichnet in seinem klassischen Text zum wissenschaftlichen Ethos die Verpflichtung zur kritischen Überprüfung von Wahrheitsansprüchen als institutionalisierte Norm des organisierten Skeptizismus und meint damit das methodologische und institutionelle Mandat, Wahrheitsansprüche nach empirischen und logischen Kriterien zu prüfen.

27 Engers, Gans 1998, S. 1348.

28 Man denke hier beispielsweise an die großen Musiklabels. Diese nehmen Künstler unter Vertrag und beschränken sich häufig auf den Vertrieb und die Bewerbung des entsprechenden Produkts. Anders verhält es sich zum Teil bei Zeitungen, beim Radio und beim Fernsehen. Hier sind die eigenen Redakteure oft sehr weitreichend an der Produktion von Inhalten beteiligt. 
den, wenn den spezifischen Kommunikationsanforderungen der Wissenschaft entsprochen wird. Typisch ist für Wissenschaftsverlage eine »fremdreferentiell finalisierte wirtschaftliche Rationalität «, ${ }^{29}$ bei der zwar die ökonomische Rationalität für gewöhnlich die grundlegende Orientierung bildet, die wissenschaftliche Rationalität aber in mehr oder minder großem Umfang in das Entscheiden von Verlagsakteuren eingeht. Schimank und Volkmann gehen nicht davon aus, dass ein echtes Konkurrenzverhältnis zwischen den beiden Orientierungen besteht und eine stärkere Berücksichtung der wissenschaftlichen Rationalität im Sinne eines Nullsummenspiels zugleich zu einem Weniger an ökonomischer Rationalität führen muss. Vielmehr können wissenschaftliche und ökonomische Rationalität in ihrer Bedeutung für das Handeln von Verlagen variieren, und es finden sich durchaus Beispiele, bei denen ökonomische und wissenschaftliche Rationalität jeweils eine starke oder jeweils eine schwache Rolle spielen. Nach Schimank und Volkmann wird die wissenschaftliche Rationalität in kleineren, häufig inhabergeführten Verlagen vergleichsweise stark berücksichtigt, während in großen Wissenschaftsverlagen im STM-Bereich (Science, Technology, and Medicine) eine vergleichsweise starke Orientierung an ökonomischer Rationalität vorherrscht. Starke Orientierung an ökonomischer Rationalität zeigt sich dabei an einer Personalstruktur, in der betriebswirtschaftliche Kompetenzen bei der Leitung stark verankert sind, in einer Programmstruktur, in der sich die Anforderung findet, dass jedes einzelne Verlagsprodukt Gewinn abzuwerfen hat, ${ }^{30}$ und in der Kommunikationsstruktur, wenn Lektoren und Herausgeber von der Controlling- oder Vertriebsabteilung regelmäßig über den Markterfolg unterrichtet werden. ${ }^{31}$

Betrachtet man die Akteurskonstellation dieser Wertschöpfungskette unter dem Gesichtspunkt des Austausches, lässt sich die Beziehung wie folgt charakterisieren: Wissenschaftler tauschen im Zuge des Produktionsprozesses die Nutzungsrechte an einem Werk gegen die Chance des Reputationserwerbs. An diesen Tausch schließt sich ein Vorgang der Kommodifikation an, wenn der Zugang zum Text durch den Verlag in Form einer Ware verkauft wird. ${ }^{32}$ Mit Blick auf das Prinzip des organisierten Skeptizismus und die Rolle der Gutachter lässt sich feststellen, dass beides sehr gut zu dem Geschäftsmodell der Verlage passt. Gutachter erbringen ihre Leis-

29 Schimank, Volkmann 2012, S. 170.

30 Eine Gewinnorientierung ist in sämtlichen Verlagen anzutreffen. Jedoch unterscheiden sich Verlage darin, ob in Bezug auf sämtliche Produkte eine Gewinnerwartung existiert oder ob es auch Publikationen gibt, die durch andere Produkte des Verlags subventioniert werden.

31 Schimank, Volkmann 2012, S. 174.

32 Hanekop, Wittke 2006, S. 212. Dies gilt natürlich nur für das übliche Subskriptionsmodell, bei dem der Verlag den Zugang zu wissenschaftlicher Literatur gegen die Zahlung einer Lizenzgebühr gewährt. In der Regel ist das vertriebene Produkt nicht der einzelne Text, sondern eine definierte Menge von Texten, zum Beispiel eine Zeitschrift oder Zeitschriftenpakete. 
tungen in der Regel unentgeltlich, ${ }^{33}$ und sie liefern einen Beitrag zur Steigerung der Qualität, was die Absatzchance einer Zeitschrift erhöht. Trotzdem liegt zwischen der wissenschaftlichen Gemeinschaft und dem Verlag keine einseitige Leistungsbeziehung vor: Verlage erbringen ebenfalls Leistungen für die Wissenschaft, indem sie Themen und Schwerpunkte in der wissenschaftlichen Kommunikation setzen ${ }^{34}$ und damit den Wissenschaftlern ein vergleichsweise verlässliches System zur Verbreitung von Forschungsergebnissen und zum Erwerb von Reputation bereitstellen. ${ }^{35}$ Neben dieser nicht geringzuschätzenden Leistung übernehmen Verlage die technische Verarbeitung des Textes über den Satz bis hin zum Druck, stellen zum Teil ein Korrektorat bereit, produzieren Metadaten für Publikationen und bedienen Vertriebsstrukturen, die es garantieren, dass die Veröffentlichungen auch von Lesern aufgefunden werden.

\subsection{Wissenschaftler als Leser: Bibliotheksvermittelte Nachfrage}

Am Ende der Werkschöpfungskette steht der Vertrieb, der die Leserschaft beliefert. Eine Besonderheit dieses letzten Glieds der Kette besteht darin, dass die Leserinnen und Leser, also ökonomisch gesprochen die Konsumenten des Produkts »Publikation «, zumeist zur selben Gruppe von Akteuren gehören wie der Autor: Es sind in der Regel beides Wissenschaftler, Wissenschaftsaffine und Wissenschaftsinteressierte. Sie treten nun allerdings nicht selbst als Nachfrager nach Publikationen auf. Zwischen den Verlagen als Anbieter von Literatur und den an der Literatur interessierten Wissenschaftlern stehen in der Regel die Bibliotheken. Indem sie die Literatur sämtlichen Bibliotheksbenutzern zugänglich machen, sorgen sie für die Aufhebung der Knappheit des Zugangs zu Publikationen und verwandeln die Literatur in ein öffentliches Gut. Hanekop und Wittke sprechen hier von einem Vorgang der De-Kommodifizierung ${ }^{36}$ - aus einem Tauschwert wird wieder ein Gebrauchswert. Die Schwierigkeit, vor der Bibliotheken bei der Herstellung des Zugangs zu wissenschaftlicher Literatur stehen, liegt darin, dass sie aufgrund der hohen Kosten für wissenschaftliche Zeitschriften eine Auswahl treffen müssen und die Qualität des

33 Der weit überwiegende Teil der Begutachtungsleistung wird unbezahlt erbracht (Rowland 2002, S. 252). In der Literatur finden sich nur wenig Belege für eine Bezahlung von Gutachtern, wobei die berichteten Anteile bei fünf Prozent der Journale liegen (Ware 2008, S. 9). Interessanterweise ist der Anteil der Journale, die den Gutachtern eine Bezahlung anbieten, in den Sozial- und Geisteswissenschaften mit neun Prozent am höchsten (ebd.).

34 Beispielsweise durch die Gründung von Zeitschriften mit spezifischen thematischen Profilen oder dem Verlegen von Monographiereihen.

35 Hanekop, Wittke 2006 , S. 210.

36 Ebd., S. 212. 
Produkts und die Notwendigkeit der Herstellung eines Zugangs nur indirekt ${ }^{37}$ und häufig erst ex post ${ }^{38}$ eingeschätzt werden können.

\section{Editorial Manager und die Produktion wissenschaftlicher Journale}

Seitens der großen Wissenschaftsverlage wurden in diese eben skizzierte Wertschöpfungskette in den vergangenen Jahren Online Editorial Management-Systeme eingeführt. Im Fall von Minerva handelt es sich um das System Editorial Manager ${ }^{39}$, das seit dem Wechsel der Herausgeberschaft zu Peter Weingart im Jahre 2007 genutzt wird. ${ }^{40}$ Bei einem Online Editorial Management-System handelt es sich um ein Softwaresystem, das auf den Servern des Verlags installiert ist und von diesem verwaltet wird. Es organisiert den gesamten Publikationsprozess von der Einreichung von Artikeln bis hin zu deren Veröffentlichung: Sämtliche Korrespondenz zwischen allen beteiligten Akteuren wird über diese Plattform abgewickelt, alle Dokumente werden vom System archiviert, und die Kommunikation wird (halb)automatisch unterstützt.

Über die Gründe des Verlags, sich stark für die Einführung dieses Systems zu engagieren, lässt sich nur spekulieren. Vordergründig mögen sie - angetrieben von einer Dienstleistungsorientierung - den Herausgebern ein Softwaresystem an die Hand geben wollen, das es ihnen ermöglicht, den editorischen Prozess ökonomisch effizient zu gestalten - denn jeder Zeitverzug, an welcher Stelle auch immer, kostet Geld, da Satz, Herstellung, Druck und Vertrieb zeitlich streng eingetaktet sind.

37 Mit bibliometrischen Indikatoren wie dem Journal Impact Factor (JIF) besteht die Möglichkeit einer solchen indirekten Bewertung der Qualität wissenschaftlicher Journale (vgl. Garfield 2006). Eine Schwierigkeit dieses Zitationsmaßes liegt darin, dass die wissenschaftlichen Disziplinen in der dem Indikator zugrunde liegenden Zitationsdatenbank unterschiedlich gut repräsentiert sind. Aus diesem Grund sollten Impact-Faktoren von Zeitschriften verschiedener Fächer nicht miteinander verglichen werden. Zudem liegen wegen der verschieden guten Repräsentation der Fächer gerade im Bereich der Geistesund Sozialwissenschaften nur für einen vergleichsweise geringen Teil der Zeitschriften Impact-Faktoren vor.

38 Neben dem JIF wird die Relevanz von Zeitschriften anhand von Ausleihstatistiken (Umlauf 1994) und Zugriffsstatistiken gemessen. Für beide Formen der Messung gilt, dass die Relevanz einer Zeitschrift erst nach dem Erwerb des entsprechenden Zugangs bestimmt werden kann.

39 Neben Editorial Manager gibt es noch das Online Editorial Management-System Open Journal Systems, das im Umfeld von Open Access entstanden ist und insbesondere von Open Access-Journalen eingesetzt wird (siehe http://pkp.sfu.ca/?q=ojs; Zugriff vom 03.04.2012). Die wesentlichen Befunde dieses Beitrags lassen sich nicht auf Open Journal Systems übertragen, da dieses System typischerweise von den Herausgebern wissenschaftlicher Journale administriert wird. Dementsprechend finden sich in diesen Fällen auch nicht die weiter unten analysierten Beobachtungskonstellationen und die starke Position des Verlags, der den Zugriff auf die vom System produzierten Prozessinformationen reguliert.

40 Die Implementation für Minerva findet sich unter http://www.editorialmanager.com/ minerva (Zugriff vom 03.04.2012). 
Ausgehend von den Überlegungen zum Produktionsprozess wissenschaftlicher Journale, lässt sich daneben die folgende Problemlage ausmachen, vor der Verlage stehen und die ein starkes Motiv für die Einführung abgibt: Wie gezeigt, sind Verlage bei der Produktion von Zeitschriften in mehrfacher Hinsicht auf die Kooperation mit Wissenschaftlern angewiesen. Deren Leistungserbringung ist zwar freiwillig und erfolgt, verglichen mit anderen freiwilligen Leistungen, vergleichsweise zuverlässig, da sie durch die oben beschriebenen Orientierungen und internen Normen abgestützt wird. Die Schwierigkeit, vor der Verlage allerdings stehen, liegt in ihren sehr beschränkten Möglichkeiten, die Erbringung und die Qualität der Leistungen von Wissenschaftlern kontrollieren zu können. Grund dafür ist, dass die Wissenschaftler nicht Teil der Organisation »Verlag « sind, in den überwiegenden Fällen keine Ressourcen erhalten (die bei Nichterbringung von Leistungen entzogen werden könnten) und nur zum Teil und nur in einem sehr eingeschränkten Sinne vertraglich gebunden sind. ${ }^{41}$ Für Unternehmen, deren Geschäft elementar von der dauerhaften Erbringung freiwilliger Leistungen Dritter abhängig ist, dürfte sich diese Lage als problematisch darstellen. Ein Motiv für die Einführung von Online Editorial Management-Systemen mag daher im Interesse der Verlage liegen, die Leistungserbringung seitens der wissenschaftlichen Akteure stärker beobachten und kontrollieren zu können. Im Folgenden möchte ich das System näher vorstellen, indem ich es anhand des Beispiels Minerva mit Blick auf zwei analytisch trennbare, aber empirisch zusammenhängende Aspekte untersuche: Editorial Manager als Mittel der Arbeitsorganisation und als Kontrollsystem.

\subsection{Editorial Manager als Mittel zur Arbeitsorganisation}

\section{Internationale Arbeitsteilung}

In arbeitsorganisatorischer Hinsicht fällt zunächst auf, dass das System darauf zielt, sämtliche Kommunikationsprozesse auf der Plattform zu konzentrieren. Dies gilt für (a) die Korrespondenz mit den Autoren, (b) die Kommunikation mit den Gutachtern, (c) die Zusammenarbeit mit dem muttersprachlichen Lektor und (d) die Kooperation mit den die Publikation weiterverarbeitenden Abteilungen von Springer, vor allem der Setzerei. Die Zentralisierung der Kommunikation auf einem mit dem Internet verbundenen Server und das Vorliegen sämtlicher Dokumente und sämtlicher Kommunikation in elektronischer Form führen zu einer Wählbarkeit in Bezug auf den Ort, an dem einzelne Produktionsschritte geleistet werden. Es wird möglich, von jedem mit dem Internet verbundenen PC auf das System zuzugreifen und an einzelnen Schritten mitzuwirken. Seinen Ausdruck findet dies an der räumlichen Verteilung der an der Produktion beteiligten Akteure:

41 Dies gilt auch mit Blick auf die Verträge zwischen Wissenschaftsverlagen und den Herausgebern wissenschaftlicher Journale. Diese spezifizieren in der Regel zwar die von den Vertragspartnern zu erbringenden Leistungen, sehen aber meist keine Sanktionen für den Fall der Nichterbringung vor. 
- Das für die Zeitschrift zuständige Management von Springer ist in Dordrecht (Niederlande) beheimatet.

- Die Redaktion mit dem Herausgeber (Editor), dem geschäftsführenden Redakteur (Managing Editor) sowie dem auftragsabhängig vom Verlag entlohnten Lektor (Language Editor) hat ihren Sitz in Bielefeld. ${ }^{42}$

- Das muttersprachliche Lektorat hatte ursprünglich seinen Sitz in Großbritannien. Nach dem Auftreten von Qualitätsproblemen wurde die Funktion nach Bielefeld verlagert.

- Die Setzerei befindet sich in Indien.

- Die Autoren des Hefts 3/2010 kommen aus den USA, den Niederlanden, Österreich, Kanada und Japan.

- Die für die Tätigkeit unbezahlt arbeitenden Gutachter desselben Hefts stammen aus Deutschland, den USA, der Schweiz und Großbritannien.

Die relative Unabhängigkeit von einem festgelegten Ort erlaubt es dem Verlag, die für ihn Kosten erzeugenden Arbeitsprozesse unter dem Gesichtspunkt der Kostenminimierung zu reorganisieren. Dies zeigt sich bereits an relativ kleinen Arbeitsschritten, wie zum Beispiel dem Satz oder der technischen Administration des Systems Editorial Manager, die aus dem Verlagshaus in Dordrecht ausgegliedert und in andere Teile der Welt verlagert wurden, in denen das Lohnniveau geringer ist.

Den beteiligten Wissenschaftlern fordert die Arbeitsteilung auf der Grundlage von Editorial Manager erhebliche Anpassungsleistungen ab, die sich für Autoren beispielsweise wie folgt darstellen: Bevor diese bei der Redaktion ein Manuskript einreichen können, müssen sie sich zunächst beim System anmelden und einen Benutzeraccount generieren. Bei der Manuskriptübermittlung müssen Anforderungen an Dateiformate erfüllt werden, Metadaten geliefert, das Papier thematisch gemäß einem vorgegebenen Schema klassifiziert sowie Schlagworte angegeben werden. Dieser insgesamt auf 16 einzelne Webseiten verteilte Prozess führt häufig zu Schwierigkeiten, die dann meist per E-Mail geklärt werden und oft zu Lösungen führen, die außerhalb des Systems liegen.

Ein weiteres Beispiel für eine Anpassungsleistung bildet die Bezeichnung von Manuskripten durch das System. Autoren, Gutachter und Herausgeber müssen sich daran gewöhnen, dass ein Manuskript in den über das System versandten E-Mails nicht wie in der Wissenschaft üblich durch Titel oder Autorenname identifiziert wird, sondern anhand eines Codes. Dieser setzte sich bislang aus einem vierstelligen Kürzel für die Zeitschrift und einer fortlaufenden Nummer zusammen (zum Beispiel MINE285). Aufgrund des Ziels, einheitliche Codes für Manuskripteinreichungen in sämtlichen Zeitschriften von Springer zu entwickeln, hat der Verlag ein neues Code-Schema eingeführt: XXXX-D-YY-NNNNN, wobei die ersten vier Ziffern einen Teil der URL der Webseite von Editorial Manager wiederholen, »D « für »default « steht, »YY « das Jahr der Manuskripteinreichung wiedergibt und »NNNNN « eine fünfstellige fortlaufende Nummer ist, die zum Zeitpunkt der Einreichung eines Manuskripts vergeben wird. Eine solches Schema der Manuskriptbezeichnung, das

42 Leider verbietet der Vertrag zwischen Editor und Verlag - das sogenannte Publishing Agreement - die Veröffentlichung des jährlichen Budgets der Zeitschriftenredaktion. 
Kürzel wie »MINE-D-12-00319 « erzeugt, entspricht sicherlich nicht den Kommunikationsgewohnheiten der Wissenschaftler, sondern orientiert sich primär an den Anforderungen des Produktionsprozesses innerhalb des Verlags.

\section{Starke Strukturierung des Produktionsprozesses}

Wesentliches Merkmal der durch Editorial Manager geschaffenen Arbeitsorganisation ist die Zerlegung des Produktionsprozesses in einzelne Schritte, die elektronisch schematisiert werden. Solche Schritte sind beispielsweise: die automatische Information des Herausgebers darüber, dass ein neues Manuskript eingegangen ist, die Einladung von Kolleginnen und Kollegen (»Peers«) zur Begutachtung des Manuskripts, gegebenenfalls die Erinnerung eines potentiellen Gutachters daran, dass er eingeladen wurde, aber noch nicht geantwortet hat, die Information an den Herausgeber, dass ein Gutachter sich bereiterklärt hat, die Begutachtung zu übernehmen (oder, sofern er abgelehnt oder nicht geantwortet hat, dass der Herausgeber nun einen neuen Gutachter benennen soll) usw. Die elektronische Strukturierung der einzelnen Schritte kann dabei unterschiedliche Formen annehmen. Auf der einen Seite des Spektrums finden sich vollständig automatisierte E-Mails: Sämtliche Nachrichten, die den Charakter von Erinnerungen an bevorstehende Aufgaben haben oder die über einen Vorgang informieren, werden vom System ohne die Mitwirkung einer Person versandt. Auf der anderen Seite des Spektrums finden sich Nachrichten, die editiert und angepasst werden können, die das System aber durch textliche Vorlagen und Textbausteine nach Möglichkeit vorgibt: Ein Beispiel hierfür sind Briefe des Herausgebers an die Autoren, in denen die Entscheidung über Annahme oder Ablehnung eines Manuskripts mitgeteilt wird. Das System liefert eine Textvorlage für das Anschreiben; im Fall der Entscheidung »Revision « oder »Annahme zur Publikation « werden technische Hinweise gegeben, wie der Autor nun zu verfahren hat, und die Gutachten werden automatisch beigefügt.

Mit dieser Strukturierung des Arbeitsablaufs sowie der automatischen und teilautomatischen Rahmung wirkt das System standardisierend auf den Produktionsprozess. Zum einen nimmt das System den Herausgebern die Entscheidung, welcher Schritt als nächstes zu erfolgen hat, zu einem guten Stück aus der Hand. Das System realisiert dies, indem die Manuskripte im Zuge des Produktionsprozesses durch unterschiedliche Ordner wandern und diese Ordner dem Manuskript einen bestimmten Status zuweisen. In Abhängigkeit vom jeweiligen Status werden bestimmte Schritte als möglich definiert (und andere ausgeschlossen). Zum Beispiel enthält der Ordner »New Assignments «:

"... submissions that have been assigned to the Editor. They require one of the following: another Editor assignment, Reviewer invitations, or Decision. «;

oder der Ordner »Submissions with One or More Late Reviews«:

»These submissions require one of the following actions: 1) Invite Additional Reviewer(s);

2) Allow current Reviewers to complete their work; 3) Make a Decision." 
Die vom System als zulässig definierten Schritte werden durch Links aufgerufen, und das Schema wird aktiviert. Ein Beispiel für eine halbunterstützte Aktion ist die Einladung von Gutachtern. Standardmäßig ruft das System die folgende E-Mail auf, die aus dem Datensatz des jeweiligen Manuskripts bestimmte Informationen extrahiert und automatisch in die E-Mail einbaut:

\footnotetext{
»Dear \%REALNAME\%,

in view of your expertise I would be very grateful if you could review the following manuscript which has been submitted to \%JOURNALFULLTITLE\%.

Manuscript Number: \%MS_NUMBER\%

Title: \%ARTICLE_TITLE\%

Abstract: \%ABSTRACT\%

In case you accept to review this submission please click on this link:

$\%$ ACCEPT_REVIEW_INVITATION\%

If you do not have time to do this, or do not feel qualified, please click on this link:

$\%$ DECLINE_REVIEW_INVITATION\%

We hope you are willing to review the manuscript. If so, would you be so kind as to return your review to us within \%REVIEW_DAYS_TO_REVIEW\% days of agreeing to review? Thank you.

You are requested to submit your review online by using the Editorial Manager system which can be found at: \%JOURNAL_URL\%. Your username is: \%USERNAME\% and your password is: \%PASSWORD \%.
}

\section{IN ORDER TO KEEP DELAYS TO A MINIMUM, PLEASE ACCEPT OR DECLINE THIS ASSIGNMENT ONLINE AS SOON AS POSSIBLE!}

If you have any questions, please do not hesitate to contact us. We appreciate your assistance.

With kind regards,

$\%$ EDITOR_NAME\% \%

Der Wortlaut der E-Mail kann durch die Herausgeber verändert werden, doch birgt das Editieren des Textes gewisse Risiken: Die Links, mit denen die Einladungen angenommen oder abgelehnt werden, sollten ebenso wenig verändert werden wie die Zugangsinformationen zum System, da ansonsten der vorgegebene Ablauf des Begutachtungsprozesses gestört wird. Entweder registriert das System die Antwort des Gutachters nicht oder der Gutachter erhält nicht die notwendigen Informationen, die er benötigt, um der Anfrage nachzukommen. Interessanter als die praktischen Schwierigkeiten ist an diesen E-Mail-Vorlagen ein anderer Punkt, den ich als normativ handlungsprägende Kraft solcher Art von Vorlagen bezeichnen möchte. Handlungsprägend wirken sie aus zwei Gründen: Zum einen simplifizieren sie eine Entscheidungssituation. An die Stelle der ungleich aufwändigeren Frage, wie in einer bestimmten Situation zu verfahren ist, tritt die schlichte Frage, ob einem bestimmten vom System präsentierten Handlungsvorschlag gefolgt wird oder nicht. Die Vorlage entlastet damit von der Suche nach einer Handlungsmöglichkeit und ihrer Alternativen sowie von der Abwägung zwischen den verschiedenen Optionen. Mittelfristig kann dies zu einer Form von routinisiertem Handeln führen, wenn im Umgang mit dem System eine stabile affirmative Haltung gegenüber den Handlungsvorschlägen eingenommen wird. Zum anderen verspricht der Handlungsvorschlag einen anstrengungsarmen Handlungsvollzug: Die E-Mail ist unmittelbar verfügbar und kann sofort verschickt werden. Die Handlung ist schneller vollzogen als im Fall von erst 
zu ermittelnden Handlungsalternativen. Das System beinhaltet damit eine normative institutionelle Ordnung, die nicht nur die Rolle des einzelnen Akteurs definiert, sondern zum Teil bis in die einzelne Handlung hinein detaillierte Vorgaben macht, wie ein Akteur seine Rolle im Produktionsprozess wahrnehmen sollte.

Die im System steckenden Prinzipien der Zerlegung des Produktionsprozesses in einzelne aufeinander folgende Glieder und die Standardisierung von in bestimmten Situationen möglichen Handlungsoptionen tragen ein Problem in sich. Es besteht darin, dass sich im Verlauf der herausgeberischen Arbeit bestimmte Anforderungen stellen können, die bei der Konstruktion des Systems nicht vorgesehen wurden. Anders formuliert: Das implementierte Modell des Arbeitsablaufs - das WorkflowModell - kann zu rigide sein und über ein zu geringes Maß an Flexibilität verfügen. In der herausgeberischen Arbeit von Minerva erweist es sich beispielsweise regelmäßig als problematisch, dass Editorial Manager es nicht vorsieht, einem einzelnen Gutachter eines Manuskripts eine E-Mail zu schicken, ${ }^{43}$ wenngleich es häufig nötig ist, Rückfragen zu einem Gutachten zu stellen, individuelle Hinweise zur Begutachtung zu geben oder sich nach dem Fortgang der Arbeit zu erkundigen. In solchen Situationen zwingt Editorial Manager dazu, den Kontakt außerhalb des Systems zu suchen. Dies führt dann aber dazu, dass die Kommunikation von Editorial Manager nicht aufgezeichnet wird und die durch die Archivierung der Kommunikation vom System geschaffene Transparenz zumindest zum Teil verlorengeht. ${ }^{44}$

Die oben zitierte Einladung zur Begutachtung weist noch einen weiteren Aspekt auf, der für das System charakteristisch ist. Neben der Zerlegung des Prozesses in einzelne Schritte und der Präformierung von Handeln definiert das System zeitliche Vorgaben für die Erledigung der einzelnen Schritte. Gutachter sind gehalten, innerhalb von zehn Tagen auf die Einladung zu reagieren. Sofern das nicht geschieht, werden sie vom System aus dem Begutachtungsprozess entfernt, und die Herausgeber werden aufgefordert, eine andere Person zu benennen. Weitere Beispiele für durch das System definierte Zeitvorgaben ist der Begutachtungszeitraum (dieser beträgt 42 Tage), die Zeit, die ein Autor für die Überarbeitung zur Verfügung hat, oder die maximale Zeitdauer, die zur Prüfung der Druckfahnen für Autoren und Herausgeber zur Verfügung steht (48 Stunden). Werden diese Zeiträume überschritten, löst dies im System Aktionen aus: Die Gutachter, Autoren oder Herausgeber werden an die Vorgaben erinnert und zu einer baldigen Bearbeitung der Aufgabe aufgefordert. Ähnliche Erinnerungen gibt es für den Herausgeber, Gutachter innerhalb einer bestimmten Frist einzuladen oder Entscheidungen über die Annahme eines Manuskripts zu treffen.

43 Vorgesehen ist ausschließlich die Option, dieselbe E-Mail an sämtliche Gutachter eines Manuskripts zu versenden.

44 Problematisch ist dabei weniger der Umstand, dass eine einzelne E-Mail nicht im System archiviert wird, sondern vielmehr, dass mit der Möglichkeit gerechnet werden muss, dass sich Teile der Korrespondenz mit wichtigen Entscheidungen außerhalb des Systems befinden. 


\section{Zusammenfassung: Betriebliche Arbeitsorganisation innerhalb der wissenschaftlichen Gemeinschaft}

In der Zusammenschau der mit Editorial Manager eingeführten arbeitsorganisatorischen Charakteristika - (a) Einführung einer internationalen Arbeitsteilung, (b) kleinteilige Zerlegung des Produktionsprozesses, (c) Vorstrukturierung und Standardisierung des Arbeitsablaufs und (d) Definition von Zeitvorgaben - fällt zunächst ins Auge, dass diese Merkmale Teil einer betrieblichen Arbeitsorganisation sind, die üblicherweise mit ökonomischer Rationalität assoziiert wird. Die dem System zugrunde liegenden Arbeitsorganisationsprinzipien laufen darauf hinaus, den Produktionsprozess so zu gestalten, dass er effizient, wiederholbar und berechenbar wird. Denkt man nun aber an den sozialen Bereich, in dem diese Form von Arbeitsorganisation implementiert wird, gelangt man zu einem überraschenden Ergebnis: Die genannten Merkmale sind typisch für die Produktionsprozesse innerhalb von Unternehmen, nicht aber für kooperative Prozesse, die freiwillig und überwiegend unbezahlt stattfinden. An der Produktion wissenschaftlicher Journale sind nun nicht nur Mitglieder der Organisation »Verlag « beteiligt, sondern auch Wissenschaftler, die gerade keine Mitglieder des Unternehmens sind, sondern zur Umwelt von Verlagen zählen. Daher lässt sich davon sprechen, dass für Unternehmen typische Prinzipien der Arbeitsorganisation über die Grenzen des Verlags ausgedehnt und in die wissenschaftliche Community hineinkopiert werden. Editorial Manager bildet dabei das Mittel, um eine solche Ausweitung der Arbeitsorganisation auf Akteure in der Umwelt des Verlags zu erreichen. ${ }^{45}$

Von Bedeutung ist die sich hier unmittelbar anschließende Frage, ob, und wenn ja, an welcher Stelle und für wen durch die Benutzung von Editorial Manager Effizienzgewinne entstehen und ob es Akteure gibt, die eventuell die Lasten von Mehrarbeit zu tragen haben. Auf der Grundlage meiner Beobachtungen kann diese Frage nicht abschließend beantwortet werden. ${ }^{46}$ Sie geben aber einige Hinweise auf Arbeitsverteilungseffekte des Systems: Auf Seiten des Verlags entstehen zwar neue Aufgaben der Bereitstellung und des Betriebs von Editorial Manager. Große Effizienzgewinne dürften sich aber dadurch ergeben, dass die Mitarbeiter des Verlags auf standardisierten Kommunikationskanälen und in standardisierter Form mit den am Produktionsprozess beteiligten Wissenschaftlern in Kontakt stehen und in einem

45 Die Einbindung von Akteuren aus der Unternehmensumwelt ist aus der Literatur zum »Prosumer « bekannt. Kennzeichen des »arbeitenden Kunden « (Voß, Rieder 2005) ist, dass der Käufer einer Ware oder Dienstleistung in systematischer Weise in deren Produktion eingebunden wird. Im Fall von wissenschaftlichen Fachzeitschriften geht die Einbindung allerdings noch weiter: Hier werden Akteure in die Produktion mit einbezogen, die keinen unmittelbaren persönlichen Nutzen vom hergestellten Produkt haben.

46 Die Beantwortung dieser Frage würde es erfordern, den Arbeitsumfang von Autoren, Gutachtern und Redaktionen sowie Verlagen systematisch an den Fällen von zwei Gruppen von Zeitschriften zu vergleichen: solche, die ein Online Editorial Management-System einsetzen, und solche, die auf den Einsatz eines solchen Systems verzichten. Es wäre zu vermuten, dass sich in der zweiten Gruppe von Zeitschriften recht unterschiedliche Formen der Arbeitsorganisation finden.

Leviathan, 40. Jg., 2/2012 
vordefinierten Format und unter Einhaltung von Kriterien der Manuskriptgestaltung Zwischenprodukte (eben die begutachteten, geprüften und redigierten Manuskripte) erhalten, die unmittelbar weiterverarbeitet werden können. In der Redaktion wirkt das System ambivalent. Indem es den Arbeitsprozess strukturiert und die Korrespondenz zwischen den Beteiligten archiviert und verwaltet, schafft es einen nicht geringzuschätzenden Überblick über die laufenden und abgeschlossenen Begutachtungsvorgänge und deren (Zwischen-)Ergebnisse. Zudem wird die Korrespondenz automatisch oder halbautomatisch mit Textvorlagen unterstützt. Dem steht allerdings ein Kommunikationsaufwand entgegen, der seine Ursachen einerseits in den Unzulänglichkeiten des Systems (Stichwort "mangelnde Flexibilität im Workflow-Modell«) und andererseits im zum Teil geringen Erfahrungsstand im Umgang mit solchen Systemen bei Autoren und Gutachtern hat. Es wäre dabei zu vermuten, dass sowohl die Zahl der eingereichten Manuskripte bei einer Zeitschrift als auch der Umfang der Verbreitung von Online Editorial Management-Systemen innerhalb der jeweiligen Fachgemeinschaft Faktoren sind, die darauf Einfluss haben, ob für die Redaktion unter dem Strich Effizienzgewinne entstehen. Mehrarbeit ist vor allem bei den Gutachtern und Autoren zu erwarten. Neben den angesprochenen Anpassungsleistungen haben diese zusätzliche technische Anforderungen bei der Übermittlung von Manuskripten und Gutachten zu berücksichtigen, denen, soweit ich sehen kann, keine Arbeitserleichterungen gegenüberstehen.

\subsection{Editorial Manager als Kontrollsystem}

Die zweite Dimension, anhand derer ich Editorial Manager analysieren möchte, ist die Kontrolldimension. Kontrolle ist immer nur auf der Basis von Informationen möglich. ${ }^{47}$ Daher ist an dieser Stelle die Frage leitend, welche Akteure über einen Zugang zu welcher Art von Prozessinformationen verfügen und welche Art von Beobachtungskonstellation durch die Informationsverteilung eingerichtet wird.

\section{Protokollierung des gesamten Prozesses - Kontrolle des einzelnen Vorgangs}

Eine wesentliche Eigenschaft von Editorial Manager ist die Protokollierung sämtlicher Aktivitäten auf der Plattform. Nicht nur wird die Korrespondenz zwischen den Beteiligten archiviert, sondern daneben wird auch der Zeitpunkt der jeweiligen Aktivität festgehalten. Wann wurde wer zur Begutachtung von welchem Manuskript

47 Dieser Artikel beschränkt sich auf die Analyse der Bedeutung der von Editorial Manager gesammelten Informationen für den Produktionsprozess einer Zeitschrift. Die Informationen werden vom Verlag allerdings auch zu weiterreichenden Zwecken genutzt. Beispielsweise werden sie verwendet, um Wissenschaftler gezielt anzusprechen, für andere Journale im Zeitschriftenprogramm des Verlags zu schreiben. Ebenfalls nicht weiter untersucht werden kann in Rahmen dieses Beitrags, welche Risiken damit verbunden sind, dass der Verlag durch Editorial Manager einen Zugang zu vertraulichen Dokumenten gewinnt, die im Rahmen von Begutachtungsprozessen in sämtlichen Zeitschriften seines Portfolios entstanden sind. 
eingeladen, wann hat die betreffende Person auf die Einladung reagiert, wann wurde welches Gutachten geliefert, und für welches Manuskript wurde welche herausgeberische Entscheidung getroffen? Durch die Protokollierung und Archivierung der Vorgänge wird der organisatorische Aufwand für die Herausgeber stark reduziert, indem das System jederzeit einen schnellen Zugriff auf die Korrespondenz und die verschiedenen Dokumente ermöglicht. Unter Kontrollgesichtspunkten führt die Protokollierung zu einer leichteren Nachvollziehbarkeit und innerhalb des Redaktionsteams zu einer Vergrößerung der Transparenz über laufende und abgeschlossene Prozesse. Durch die Konfrontation der empirisch zu beobachtenden Realität der einzelnen Arbeitsschritte mit den durch das System definierten normativen Vorgaben können zeitliche Verzögerungen identifiziert werden und lässt sich Handlungsbedarf feststellen. Dies kann dann beispielsweise in die Erinnerung eines Gutachters, die Einladung eines weiteren Gutachters oder die Übersendung einer Entscheidung münden.

\section{Auswertung der Prozessdaten - Informationale Unterstützung von Entscheidungen}

Das System belässt es allerdings nicht bei einer begleitenden Protokollierung, sondern nutzt die Daten, um sie in aggregierter Form auszuwerten. Dabei ist die Aggregation und Auswertung ein Vorgang, der nicht des Zutuns eines Akteurs bedarf. Er erfolgt vielmehr automatisch und in Echtzeit, so dass die erzeugten Informationen für alle weiteren Operationen des Systems zur Verfügung stehen. Ein Beispiel für eine solche Auswertung bilden Informationen über die bisherige Mitarbeit (»Performance «) von Gutachtern. Im Zuge der Einladung eines Gutachters wird dem Herausgeber eine Übersicht über die bisherigen Begutachtungsleistungen des betreffenden Wissenschaftlers gegeben, die unter anderem die folgenden Informationen umfasst: Anzahl der bisherigen Einladungen, angenommene Einladungen, Zeitpunkt der letzten Einladung und unter »average days outstanding " der durchschnittliche Zeitraum, der zwischen der Annahme der Einladung und dem Hochladen des Gutachtens auf die Plattform vergeht.

Insbesondere die letztgenannte Zahl ist hier von Interesse: Die Auswertung von Daten, die während vorangegangener Begutachtungsvorgänge gesammelt wurden, wird hier genutzt, nachfolgende Entscheidungen mit Informationen zu unterstützen. Im Spiegel dieser quantitativen Maßzahlen kann ein direkter Vergleich zwischen Gutachtern stattfinden.

\section{Partielle Information des Autors}

Allerdings ist der Herausgeber nicht der einzige Akteur, der Zugriff auf Informationen über den Prozess hat. Auch der Autor hat die Möglichkeit, sich über die Vorgänge bezüglich seines Manuskripts fortlaufend zu informieren. $\mathrm{Zu}$ diesem Zweck erhält er nach der Übermittlung des Manuskripts eine Bestätigungs-E-Mail 
mit einem Link auf eine Webseite, auf der der Stand des herausgeberischen Prozesses nachvollzogen werden kann:

»During the review process, you can keep track of the status of your manuscript by accessing the following web site:

http://minerva.edmgr.com/

Your username is: DELETED

Your password is: DELETED «

Interessant an dieser Funktion ist, dass sie zumindest partiell den Schleier des Redaktionsgeheimnisses aufhebt. Autoren sind bei wiederholtem Besuch der Seite in der Lage, nachzuvollziehen, welcher Schritt wie lange gedauert hat und - sofern es zu Verzögerungen kommt - an welcher Stelle der Prozess momentan »hängt «.

\section{Supervision durch den Verlag}

Eine Beobachtung des herausgeberischen Prozesses findet jedoch nicht nur seitens der unmittelbar beteiligten wissenschaftlichen Akteure statt. Vielmehr wertet das System die einzelnen prozessbezogenen Informationen aus und stellt sie dem Verlag in höher aggregierter Form zur Supervision des Zeitschriftenproduktionsprozesses zur Verfügung. Neben dem Verhältnis der Anzahl an Einreichungen und den akzeptierten und abgelehnten Manuskripten liefert das System auch leistungsbezogene Informationen wie zum Beispiel das Verhältnis von eingeladenen Gutachtern zu denen, die die Begutachtung eines Manuskripts übernommen haben. Zudem bereitet es prozessbezogene Informationen auf, etwa die Zeitdauer, die durchschnittlich zwischen der Einreichung und dem Druck eines Manuskripts vergeht, und die durchschnittliche Zeitdauer beispielsweise zwischen (a) der Einreichung des Manuskripts und der Einladung von Gutachtern, (b) der Einladung von Gutachtern und ihrer Antwort und (c) die Zeitdauer zwischen der Zusage, die Begutachtung zu übernehmen, und der Übersendung des Gutachtens selbst. Diese Maßzahlen werden von den zuständigen Managern von Springer genutzt, um im Rahmen von Besprechungen mit den Herausgebern die Performance des Zeitschriftenproduktionsprozesses zu diskutieren.

\section{Zusammenfassung: Wechselseitige Beobachtung und Prozesskontrolle}

Das Begutachtungssystem wissenschaftlicher Fachzeitschriften beinhaltet die Beurteilung der Arbeit eines Autors durch Herausgeber und Gutachter. Darauf beschränkt sich die Beobachtungskonstellation allerdings nicht. Stefan Hirschauer weist am Beispiel der Zeitschrift für Soziologie darauf hin, dass die Urteile von Gutachtern und Voten von Herausgebern ihrerseits von Fachkollegen innerhalb der Redaktion beobachtet werden und im Wissen um diese Beobachtung verfasst werden, ${ }^{48}$ so dass von einer komplexen Beobachtungskonstellation gesprochen werden 
muss. Durch die Einführung von Online Editorial Management-Systemen wird in diese Konstellation eine weitere Beobachtungsdimension eingeführt, die sich nicht auf Qualitätsurteile, sondern auf den Arbeitsprozess selbst bezieht: Autoren beobachten den Prozess der Bearbeitung ihrer eingereichten Manuskripte, Herausgeber die Geschwindigkeit der Gutachter bei der Anfertigung von Gutachten und die der Autoren bei der Überarbeitung von Manuskripten. Eine Supervision des gesamten Prozesses findet auf aggregierter Ebene durch die Verlage statt. Ohne Zweifel handelt es sich hier nicht um eine wechselseitige Beobachtung der Akteure "auf Augenhöhe«. Es gibt eine Zentrale, nämlich den Verlag und nicht etwa die Herausgeber, die über das Monopol vollständiger Informationen verfügt und die, das ist vielleicht noch wichtiger, darüber bestimmt, wer Zugriff auf welche Informationen erhält: Die Administration und Konfiguration des Systems liegt beim Verlag. Allerdings würde man den Charakter der Beobachtungskonstellation verkennen, würde man sie als hierarchisch auffassen und in die Nähe des Foucault'schen Panopticons rücken. Der Clou besteht gerade darin, dass sich die am Produktionsprozess beteiligten Akteure wechselseitig kontrollieren. So wird sichergestellt, dass die Akteure - immer im Wissen um die wechselseitige Beobachtung - ihren jeweiligen Aufgaben termingerecht nachkommen.

\section{Schluss: Zusammenstöße zwischen wissenschaftlicher und ökonomischer Rationalität}

In den vorangegangenen Schritten habe ich gezeigt, dass mit dem System Editorial Manager Prinzipien der Arbeitsorganisation und Prozesskontrolle implementiert wurden, die eher für Unternehmen typisch sind. Diesen wird hier über die Grenzen der Verlagsorganisation hinweg in die wissenschaftliche Gemeinschaft hinein Geltung abverlangt. Wenn aber ein solches System, das letztlich dem ökonomischen Ziel der Optimierung und der Kontrolle des Prozessablaufs dient, in einem sozialen Bereich Anwendung findet, der nach einer anderen Rationalität operiert, ist mit der Möglichkeit eines konfligierenden Aufeinandertreffens der beiden Rationalitäten zu rechnen. ${ }^{49}$ Abschließend möchte ich einige Beispiele für ein solches Aufeinandertreffen skizzieren:

- Ein erstes Beispiel für eine solche Kollision bildet die Einführung von ProzessInformationen zu den Gutachtern in die Gutachterauswahl. Werden diese Informationen von den Herausgebern bei der Wahl mit berücksichtigt, wird die in der Vergangenheit gemessene Geschwindigkeit der Begutachtung zur Grundlage weiterer Operationen. Damit verändern sich die Kriterien der Gutachter-

49 Wie weiter oben bereits ausgeführt, ist eine Kollision von Rationalitäten nur eine von mehreren logischen Möglichkeiten. Eine steigende Bedeutung ökonomischer Rationalität kann natürlich auch mit steigender, gleichbleibender und sinkender Relevanz von wissenschaftlicher Rationalität einhergehen. Die Zuspitzung auf die Kollisionen zwischen beiden Rationalitäten - mit dem Effekt einer in der Tendenz sinkenden Bedeutung wissenschaftlicher Rationalität - ist der hier verfolgten Fragestellung nach der Folgenhaftigkeit von Online Editorial Management-Systemen für die Wissenschaft geschuldet.

Leviathan, 40. Jg., 2/2012 
auswahl: $\mathrm{Zu}$ dem Kriterium »Expertise « oder »fachliche Kompetenz « tritt das der Wissenschaft strenggenommen äußerliche Kriterium »Geschwindigkeit ", das sich primär auf den Produktionsprozess bezieht. In Bezug auf dieses Kriterium liegt es nahe, kurze Zeiträume als positiv (weil funktional für den Prozess) und lange Zeiträume als negativ zu bewerten. Dies wirft Anschlussfragen bezüglich des Verhältnisses der beiden Kriterien zueinander auf, die allerdings im Rahmen dieses Aufsatzes nicht beantwortet werden können.

- Mein zweites Beispiel für solche Kollisionen bezieht sich auf automatisch generierte E-Mails, die in Fällen versandt werden, in denen Gutachter auf Einladung nicht reagieren. Mittlerweile haben wir das System so konfigurieren lassen, dass die sogenannten » un-invite messages « nicht mehr automatisch verschickt werden. Die Nachricht beinhaltet den folgenden Text:

»Dear NAME, DELETED,

Because we did not receive a reply to our request for a review of \NAME OF THE MANUSCRIPT, DELETED «, MINE1 for Minerva, we assume you must be very busy or on extended travel. We will select an alternate reviewer in order to keep reviewing delays at a minimum.

We hope that we may utilize your services in the future.

With kind regards,

The Editors at Minerva «

In Anbetracht der Tatsache, dass die Begutachtung eine Leistung darstellt, die von Wissenschaftlern unbezahlt erbracht wird, erscheint der Text wenig angemessen. Auf der Textoberfläche scheint die Nachricht korrekt zu sein; für einen Gutachter mag sie aber einen impliziten Vorwurf beinhalten, wenn seine ausbleibende Antwort auf die Anfrage der Redaktion mit zeitlichen Verzögerungen in Verbindung gebracht wird. So erscheint der nicht antwortende Wissenschaftler als ein Element in der Produktionskette, der den reibungslosen Ablauf stört. Dies wurde zumindest zum Teil von den von uns angefragten und später wieder "ausgeladenen " Gutachtern so wahrgenommen, da uns E-Mails erreichten, die rechtfertigenden Charakter hatten und darauf zielten, die ausgebliebene Antwort auf die Einladung zu begründen. Die Ursachen dafür, dass in diesen »Ausladungen " der angemessene Ton nicht getroffen wird, kann in einer starken Ausrichtung von Editorial Manager auf die Produktionsrationalität hin gesehen werden. Die zeitliche Organisation des Produktionsablaufs steht im Fokus, während nicht berücksichtigt wird, dass hier vermittels einer teilautomatisierten, initiativen Anfrage eine freiwillige Leistung nachgefragt wird.

- Ein drittes Beispiel für eine solche Kollision bilden automatisch versandte EMails, die unmittelbar nach dem Eingang eines Gutachtens an die Gutachter geschickt werden:

»Dear NAME DELETED,

Thank you very much for your review of manuscript

MINE104, , NAME OF THE MANUSCRIPT, DELETED

We greatly appreciate your assistance.

With kind regards,

Journals Editorial Office

Springer 
Dieser E-Mail liegt der Gedanke zugrunde, dass der Dank an einen Gutachter nach getaner Arbeit ein notwendiger Bestandteil des Produktionsprozesses ist. Damit wird dem Umstand der Freiwilligkeit der Begutachtungsleistung Rechnung getragen. Allerdings geschieht dies in verunglückter Weise. Erstens liegt dem Dank ein Kategorienfehler zugrunde, denn danken können nur Personen, nicht aber Computersysteme. Die unmittelbare Versendung der Nachricht und die starke Standardisierung lassen kaum einen Zweifel daran, dass es sich hier um eine automatisch generierte E-Mail handelt. Zweitens ist die Begutachtung eines Manuskripts eine fachliche Leistung, deren Würdigung voraussetzt, dass die anerkennende Person ebenfalls »vom Fach « ist. Neben dem erkennbar automatischen Ursprung der E-Mail ist daher auch der Name des Unterzeichners »schräg «. Der Dank (und damit die Anerkennung der erbrachten Leistung) findet im Namen eines Verlags und nicht im Namen des Herausgebers der Zeitschrift statt.

Meine Beispiele für Kollisionen zwischen wissenschaftlicher Rationalität und Produktionsrationalität mögen vordergründig wie kleine Verstöße gegen den guten Stil wirken. Ich denke aber, dass mehr hinter ihnen steckt. Denn: Freiwillige Leistungen von Fachkollegen sind ein Gut, das pfleglich behandelt werden sollte. Dies gilt insbesondere in Zeiten, in denen der Publikationsdruck steigt und die Begutachtung von Zeitschriftenartikeln in Konkurrenz zu einer wachsenden Zahl anderer Verpflichtungen tritt. Generell lässt sich sagen, dass durch den Einsatz von Online Editorial Management-Systemen in die Kommunikation zwischen den Mitgliedern der Fachgemeinschaft eine gewisse Distanz tritt. Für die Erbringung freiwilliger Leistungen ist dies vermutlich keine günstige Bedingung.

\section{Literatur}

Atkins, Daniel E. et al. 2003. Revolutionizing science and engineering through cyberinfrastructure: report of the National Science Foundation Blue-Ribbon Advisory Panel on cyberinfrastructure. http://www.nsf.gov/od/oci/reports/atkins.pdf (Zugriff vom 03.04.2012).

Brintzinger, Klaus-Rainer 2010. »Piraterie oder Allmende der Wissenschaft? Zum Streit um Open Access und der Rolle von Wissenschaft, Bibliothek und Markt bei der Verbreitung von Forschungsergebnissen ", in Leviathan 38, 3, S. 331-346.

Engers, Maxim; Gans, Joshua S. 1998. »Why referees are not paid (enough) «, in The American Economic Review 88, 5, S. 1341-1349.

Garfield, Eugene 2006. » The history and the meaning of the journal impact factor «, in JAMA 295, 1, S. 90-93.

Hagstrom, Warren O. 1965. The scientific community. New York, London: Basic Books.

Hanekop, Heidemarie; Wittke, Volker 2006. »Das wissenschaftliche Journal und seine möglichen Alternativen: Veränderung der Wissenschaftskommunikation durch das Internet «, in Internetökonomie der Medienbranche, hrsg. v. Hagenhoff, Svenja, S. 201-233. Göttingen: Universitätsverlag Göttingen.

Hirschauer, Stefan 2002. Die Innenwelt des Peer Review. Qualitätszuschreibung und informelle Wissenschaftskommunikation in Fachzeitschriften. Expertise im Rahmen der SciencepolicyFörderinitiative des BMBF. http://www.sciencepolicystudies.de/dok/expertise-hirschauer.pdf (Zugriff vom 03.04.2012).

Hirschauer, Stefan 2005. Publizierte Fachurteile. Lektüre und Bewertungspraxis im Peer Review, in Soziale Systeme 11, 1, S. 52-82. 
Jankowski, Nicholas 2009. »The countours and challenges of e-research ", in e-research. Transformation in scholarly practice, hrsg. v. Jankowski, Nicholas, S. 3-37. New York, London: Routledge.

Mayntz, Renate 1988. „Funktionelle Teilsysteme in der Theorie sozialer Differenzierung «, in Differenzierung und Verselbständigung. Zur Entwicklung gesellschaftlicher Teilsysteme, hrsg. v. Mayntz, Renate et al., S. 11-44. Frankfurt a. M., New York: Campus.

Merton, Robert K. 1973. "The normative structure of science ", in The sociology of science, hrsg. v. Merton, Robert K., S. 267-278. Chicago, London: The University of Chicago Press.

Nentwich, Michael 2003. Cyberscience. Research in the age of the internet. Wien: Austrian Academy of Science Press.

Peters, Douglas, P.; Ceci, Stephen J. 1982. »Peer-review practices of psychological journals: the fate of published articles, submitted again ", in Peer commentary on peer review. A case study in scientific quality control, hrsg. v. Harnad, Stevan, S. 3-11. Cambridge: Cambridge University Press.

Rowland, Fytton 2002. »The peer-review process ", in Learned Publishing 15, S. 247-258.

Schimank, Uwe 1988. "Gesellschaftliche Teilsysteme als Akteursfiktionen «, in Kölner Zeitschrift für Soziologie und Sozialpsychologie 40, 4, S. 619-639.

Schimank, Uwe 2005. Differenzierung und Integration der modernen Gesellschaft. Beiträge zur akteurszentrierten Differenzierungstheorie 1. Wiesbaden: VS Verlag für Sozialwissenschaften.

Schimank, Uwe 2007. Theorien gesellschaftlicher Differenzierung. 3. Aufl. Wiesbaden: VS Verlag für Sozialwissenschaften.

Schimank, Uwe; Volkmann Ute 2012. »Die Ware Wissenschaft: Die fremdreferentiell finalisierte wirtschaftliche Rationalität von Wissenschaftsverlagen ", in Wirtschaftliche Rationalität, hrsg. v. Engels, Anita; Knoll, Lisa, S. 165-184. Wiesbaden: VS Verlag für Sozialwissenschaften.

Springer 2009. Overview 2009. http://www.springer.com/cda/content/document/cda_downloaddocument/Overview_2009.pdf?SGWID=0-0-45-960637-0 (Zugriff vom 03.04.2012).

Umlauf, Konrad 1994. »Etatverteilung in Öffentlichen Bibliotheken - zu Bedeutung der Ausleihstatistik, der Preise und der Nicht-Buch-Medien «, in Bibliothek Forschung und Praxis 18, 3, S. 297-311.

Voß, G. Günther; Rieder, Kerstin 2005. Der arbeitende Kunde. Wenn Konsumenten zu unbezablten Mitarbeitern werden. Frankfurt a. M., New York: Campus.

Ware, Mark 2008. Peer review: benefits, perceptions and alternatives. London: Publishing Research Consortium. http://www.publishingresearch.net/documents/PRCsummary4Warefinal.pdf (Zugriff vom 03.04.2012).

Zuckermann, Harriet; Merton, Robert K. 1971. "Patterns of evaluation in science: institutionalisation, structure and functions of the referee system ", in Minerva 9, 1, S. 66-100. 
Zusammenfassung: Online Editorial Management-Systeme greifen tief in den Produktionsprozess wissenschaftlicher Journale ein und haben dort weitreichende Folgen. In arbeitsorganisatorischer Hinsicht dehnen sie betriebliche Organisationsprinzipien auf wissenschaftliche Fachgemeinschaften aus. Zudem etablieren sie ein Beobachtungsarrangement, in dem sich die Beteiligten wechselseitig kontrollieren.

Stichworte: Online Editorial Management-Systeme, wissenschaftliche Journale, Redaktion, Arbeitsorganisation, Begutachtungsverfahren

\section{Online editorial management systems and the production of academic journals}

Summary: The use of online editorial management systems significantly transforms the process of producing scientific journals. With respect to work organisation, they expand principles of commercial business and implement them within scientific communities. In addition, they give rise to a system of monitoring in which all involved keep a check on each other.

Keywords: Online editorial management systems, scholarly communication, scientific journals, academic journals, editorial office, peer review

Autor

Dr. Niels Taubert

Institut für Wissenschafts- und Technikforschung

Universität Bielefeld

Postfach 100131

33501 Bielefeld

E-Mail: ntaubert@uni-bielefeld.de

Leviathan, 40. Jg., 2/2012 https://doi.org/10.5771/0340-0425-2012-2-297 